\title{
Recording Temporal Signals with Minutes Resolution Using Enzymatic DNA Synthesis
}

Namita Bhan ${ }^{1,10, *}$, Alec Callisto1,*, Jonathan Strutz ${ }^{1}$, Joshua Glaser ${ }^{2}$, Reza Kalhor ${ }^{3}$, Edward Boyden ${ }^{4,5,6}$, George Church ${ }^{3,4}$, Konrad Kording ${ }^{2}$, Keith E.J. Tyo ${ }^{1, \ddagger}$

${ }^{1}$ Department of Chemical and Biological Engineering, Northwestern University, Evanston, IL, 60208, USA ${ }^{2}$ Center for Theoretical Neuroscience, Columbia University, New York, NY, 10027, USA

${ }^{3}$ Center for Epigenetics, Johns Hopkins School of Medicine, Baltimore, MD, 21205, USA

${ }^{4}$ Department of Brain and Cognitive Sciences, MIT, Cambridge, MA, 02139, USA

${ }^{5}$ McGovern Institute, MIT, Cambridge, MA, 02139, USA

${ }^{6}$ Howard Hughes Medical Institute, Department of Neurobiology, Harvard Medical School, Boston, MA, 02115, USA

7Department of Genetics, Harvard Medical School, Boston, MA, 02115, USA

${ }^{8}$ Wyss Institute for Biologically Inspired Engineering at Harvard University, Boston, MA, 02115, USA

${ }^{9}$ Department of Neuroscience, University of Pennsylvania, Philadelphia, PA, 19104, USA

${ }^{10}$ Mitolab, Cambridge, MA, 02139, USA

${ }^{*}$ These authors contributed equally to this work

¥Corresponding author: Keith E.J. Tyo

Telephone: +18478680319

Fax: +18474913728

Email: k-tyo@northwestern.edu

\section{Competing Financial Interests}

A utility patent application has been filed for some of the developments contained in this article by KT, NB, AC, JS, JG and KK.

Classification: Biological Sciences/Applied Biological Sciences

Keywords: DNA-based recording, enzymatic DNA synthesis, physiological signal recording, synthetic biology

This file includes:

Supplementary methods 1-6

Supplementary figures S1-S21

Supplementary tables S1-S2 
Supplementary Methods: Extended description of methods

\section{Extension reaction with individual dNTPs for testing effect of $\mathrm{Co}^{2+}$ :}

For initial testing to show $\mathrm{Co}^{2+}$ dependent dNTP preference change the ssDNA substrate used was AMD006 (Table S2). Total reaction volume was $25 \mu \mathrm{L}$ with $0.1 \mu \mathrm{M}$ ssDNA substrate, 1x NEB TdT reaction buffer, and $0.1 \mathrm{mM}$ of each dNTP tested. Final concentration of $\mathrm{CoCl}_{2}$ in the test reaction was $0.25 \mathrm{mM}$. Reactions were initiated by addition of 5 units of TdT per reaction. Reactions were run for 30 minutes at 37 ${ }^{\circ} \mathrm{C}$ and stopped by boiling at $70{ }^{\circ} \mathrm{C}$ for 10 minutes. Then, $8 \mu \mathrm{L}$ of the reaction was mixed with $12 \mu \mathrm{L}$ of TBE-Urea loading dye and boiled for 10 minutes at $100^{\circ} \mathrm{C}$. All of the diluted extension reaction was then loaded onto $30 \mu \mathrm{L}, 10$ well $10 \%$ TBE-Urea Gel (Bio-Rad) and run for 40 minutes at $200 \mathrm{~V}$. Immediately after the run was over, the gel was stained with Sybr Gold for 15 minutes and imaged on ImageQuant BioRad.

\section{Extension reactions for $1 \rightarrow 0$ set-up:}

$\mathrm{Mg}^{2+}$ only for 1 hour (signal 0 ) and $\mathrm{Mg}^{2+}+\mathrm{Co}^{2+}$ for 1 hour (signal 1 ) were set-up as regular extension reactions mentioned in Materials and Methods. The $1 \rightarrow 0$ reactions where the signal changed from 1 to 0 at 40 minutes were put through a ssDNA was step at 40 minutes. ssDNA wash to remove cations, TdT and dNTPs was done exactly as mentioned in Materials and Methods. Reactions were all run for 1 hour in triplicates. Signal 0 and signal 1 controls were run for 1 hour for each set-up in triplicates and also put through the ssDNA wash step at 40 minutes.

\section{Derivation of Equation 5}

We start by deriving the equations for the average rate before the switch $\left(r_{A}\right)$ and after the switch $\left(r_{B}\right)$ for condition, $i$ :

$$
\begin{gathered}
r_{a, i}=\frac{j_{i}^{*}}{t_{i}^{*}} \\
r_{b, i}=\frac{1-j_{i}^{*}}{t_{\text {expt }}-t_{i}^{*}}
\end{gathered}
$$

where $j_{i}^{*}$ is the average location in the sequences (length fraction, 0 to 1 ) at which the output signal, $s_{i j}$, reaches 0.5 (Equation 4), $t_{i}^{*}$ is the switch time, and $t_{\text {expt }}$ is the total duration of the experiment. Because we can estimate $r_{a, i}$ and $r_{b, i}$ from average rates of the 0 and 1 controls across replicates $\left(\overline{r_{a, c t r l}}\right.$ and $\overline{r_{b, c t r l}}$, we can use their ratio to combine equation $1 \mathrm{a}$ and $2 \mathrm{a}$, above to write

$$
\frac{\overline{r_{a, c t r l}}}{\overline{r_{b, c t r l}}} \approx \frac{r_{a, i}}{r_{b, i}}=\frac{j_{i}^{*}}{t_{i}^{*}}\left(\frac{t_{\text {expt }}-t_{i}^{*}}{1-j_{i}^{*}}\right)
$$

Solving for $t_{i}^{*}$, we get equation 5 :

$$
t_{i}^{*}=\frac{\alpha t_{\exp t}}{\frac{1}{j_{i}^{*}}+\alpha-1}
$$

where 


$$
\alpha=\frac{\overline{r_{b, c t r l}}}{\overline{r_{a, c t r l}}}
$$

We use equation 5 for time prediction $\left(t_{i}^{*}\right)$ after calculating $j_{i}^{*}$ for a given condition and $\alpha$ from the 0 and 1 controls. In equation $4 \mathrm{a}, a$ is the first condition before the switch ( 0 or 1$)$ and $b$ is the condition after the switch (1 or 0$)$.

\section{Extensions reaction set-up for calculating rate of dNTP addition:}

Each extension reaction consisted of a final concentration of $10 \mu \mathrm{M}$ initiating ssDNA substrate, $1 \mathrm{mM}$ dNTP mix (each dNTP at $1 \mathrm{mM}$ final concentration), 1.4x NEB TdT reaction buffer, and 10 units of TdT to a final volume of $50 \mu \mathrm{L}$. The ssDNA substrate used for this extension reaction was CS1_5N. We have shown (data not included) that the identity of the last 5 bases on the 3' end of the substrate affects the identity of the dNTP added to the ssDNA substrate. Thus, we purchased a ssDNA substrate (CS1_5N) with the last 5 bases having the base composition same as TdT dNTP preference under signal 0 ( $25 \%$ dATP, $15 \%$ dCTP, $45 \%$ dGTP and $15 \%$ dTTP). The reactions were initiated upon addition of TdT and run at $37^{\circ} \mathrm{C}$ for 2 hours. $2 \mu \mathrm{L}$ of sample was collected and immediately frozen (on ice, $0^{\circ} \mathrm{C}$ ) at $30 \mathrm{~s}, 1 \mathrm{~min}, 2 \mathrm{~min}, 3 \mathrm{~min}, 4 \mathrm{~min}, 5$ $\mathrm{min}, 10 \mathrm{~min}, 20 \mathrm{~min}, 30 \mathrm{~min}, 45 \mathrm{~min}, 60 \mathrm{~min}, 92 \mathrm{~min}$ and $120 \mathrm{~min}$. Subsequently, each sample was put through the ligation and Illumina library generation process as mentioned in Materials and Methods.

\section{Test set-up for checking ssDNA clean-up kit bias:}

$\mathrm{Mg}^{2+}$ only for 1 hour (signal 0 ) and $\mathrm{Mg}^{2+}+\mathrm{Co}^{2+}$ for 1 hour (signal 1 ) were set up as regular extension reactions mentioned in Materials and Methods. The $0 \rightarrow 1$ reactions where the signal changed from 0 to 1 during the 1 hour extension were run starting with $45 \mu \mathrm{L}$ with $\mathrm{Mg}^{2+}$ only. $5 \mu \mathrm{L}$ of $2.5 \mathrm{mM} \mathrm{CoCl}_{2}$ was added at $10 \mathrm{~min}$. Reactions were all run for 1 hour in triplicates. Fresh signal 0 and signal 1 controls were run for 1 hour with each set-up. $2 \mu \mathrm{L}$ of extension reaction was used for ligation ("No Wash" set of samples). Ligation and subsequent PCR steps for Illumina library generation were followed as mentioned in Materials and Methods. Rest of the $48 \mathrm{uL}$ of extension reaction was washed using the ssDNA clean-up kit. Protocol was followed as mentioned in the kit. ssDNA was eluted into $25 \mu \mathrm{L}$ of $\mathrm{ddH}_{2} \mathrm{O}$ and $2 \mu \mathrm{L}$ of that was used for ligation ("Wash" set of samples). Ligation and subsequent PCR steps for Illumina library generation were followed as mentioned in Materials and Methods. Data obtained from Illumina sequencing was analyzed for the "No Wash" and "Wash" set of samples. Further, switch time calculations were carried out as mentioned previously (Fig. S11).

\section{High calcium conditions for TURTLES-2 reactions:}

High calcium conditions for $0 \rightarrow 1$ and $1 \rightarrow 0$ reactions had different compositions to enable transitions without employing intermediate column washes. Although commercial TdT reaction buffer contains no added calcium, we observed that CaM-mTdT(M13-388) was inactivated in reactions that were not supplemented with at least $50 \mu \mathrm{M}$ EGTA (Figure S17), very likely due to calcium present in water or other reagents used. By titrating EGTA into a TdT extension reaction supplemented with $7 \mu \mathrm{M}$ fura-2 until the fura-2 signal plateaued, we estimated that most free calcium could be eliminated from the reactions by the addition of $50 \mu \mathrm{M}$ EGTA. By employing the un-supplemented reaction buffer as the high calcium condition for $1 \rightarrow 0$ reactions, we were able to transition to a low calcium condition with the addition of $50 \mu \mathrm{M}$ EGTA. 


\section{Supplementary Figures}

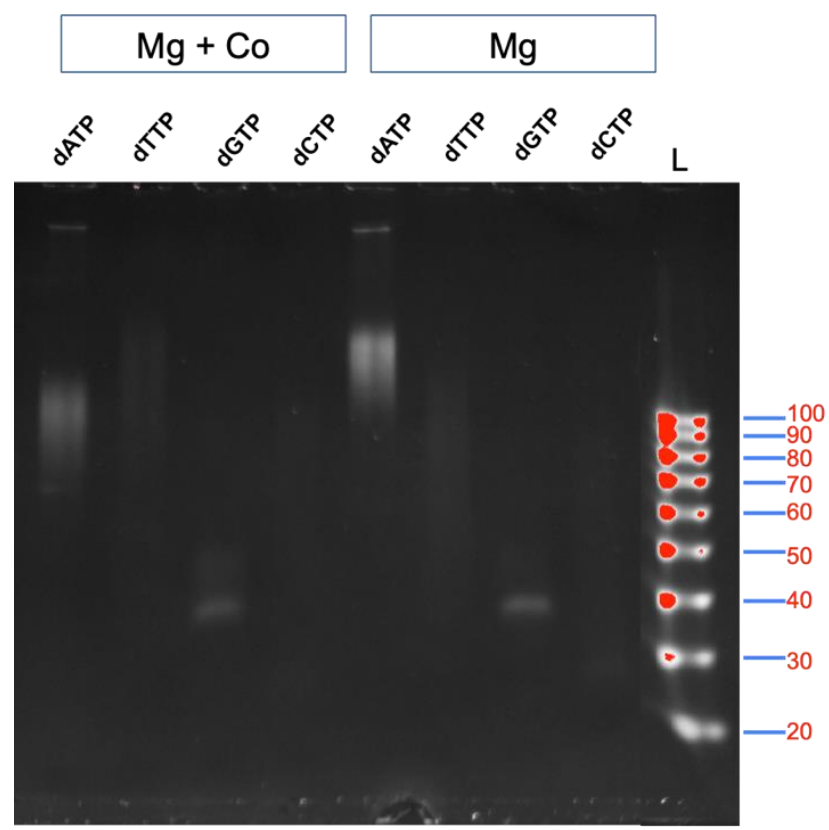

Figure S1: Testing change in individual dNTP preference upon $\mathrm{Co}^{2+}$ addition

ssDNA substrate extensions carried out by TdT using just dATP, dTTP, dGTP, or dCTP in presence of $\mathrm{Mg}^{2+}+\mathrm{Co}^{2+}$ (first 4 lanes) or in presence of just $\mathrm{Mg}^{2+}$ (next 4 lanes) were run on a gel. "L" is ssDNA size marker. Reactions were carried out as mentioned in supplementary text. 

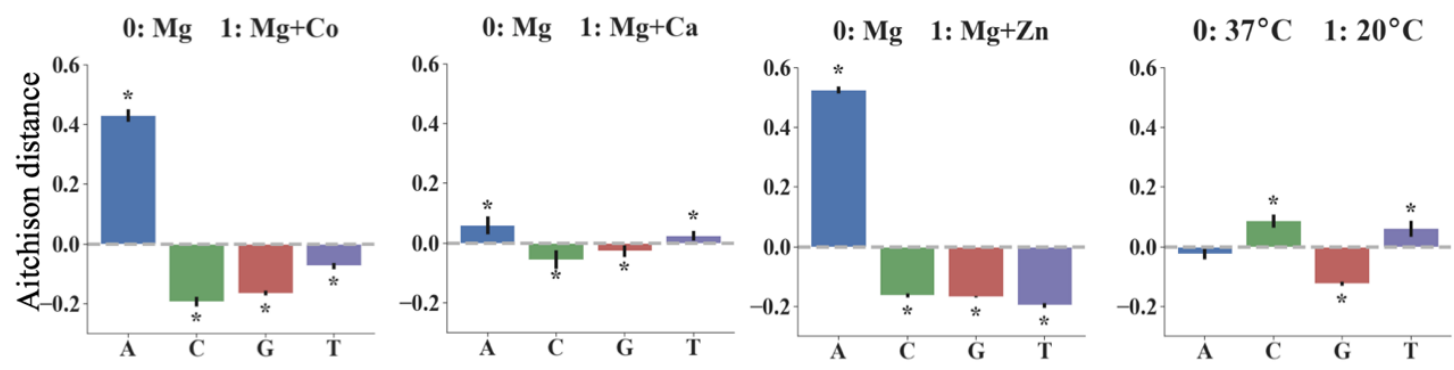

Figure S2: Aitchison distance for dATP, dCTP, dGTP and dTTP incorporation by TdT in the presence or absence of various signals. Signal 0 is always $10 \mathrm{mM} \mathrm{Mg}^{2+}$ at $37^{\circ} \mathrm{C}$ for 1 hour. Signal 1 was, going from left to right: (1) $10 \mathrm{mM} \mathrm{Mg}^{2+}+0.25 \mathrm{mM} \mathrm{Co}^{2+}$ at $37^{\circ} \mathrm{C}$ for 1 hour; (2) $10 \mathrm{mM} \mathrm{Mg}^{2+}+1 \mathrm{mM} \mathrm{Ca}^{2+}$ at 37 ${ }^{\circ} \mathrm{C}$ for 1 hour; (3) $10 \mathrm{mM} \mathrm{Mg}+20 \mu \mathrm{M} \mathrm{Zn}^{2+}$ at $37^{\circ} \mathrm{C}$ for 1 hour; and (4) $10 \mathrm{mM} \mathrm{Mg}^{2+}$ at $20^{\circ} \mathrm{C}$ for 1 hour. Error bars show two standard deviations of the mean Aitchison distance. Statistical significance was assessed after first transforming the data into Aitchison space which makes each dNTP frequency change statistically independent of the others. All base incorporation changes were found to be statistically significant, as shown by asterisks, $(\alpha=0.01)$ except for the change in dATP for $20{ }^{\circ} \mathrm{C}$ signal $(p$-value $=$ 0.019). 


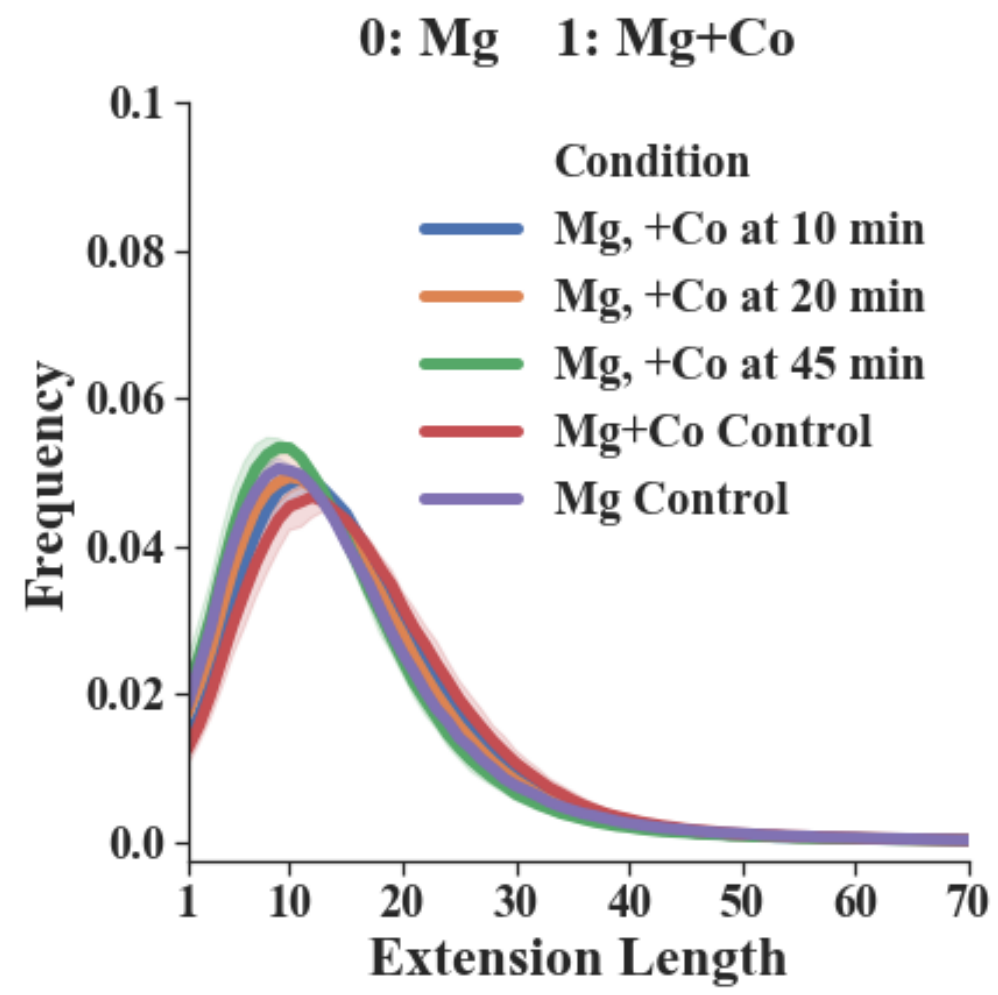

Figure S3: Length distribution of extensions upon addition of $\mathrm{Co}^{2+}$ based on NGS data.

We calculated the mean frequency distribution of extension lengths for each condition (three biological replicates for each condition). Addition of $\mathrm{Co}^{2+}$ did not change the length distribution significantly. 


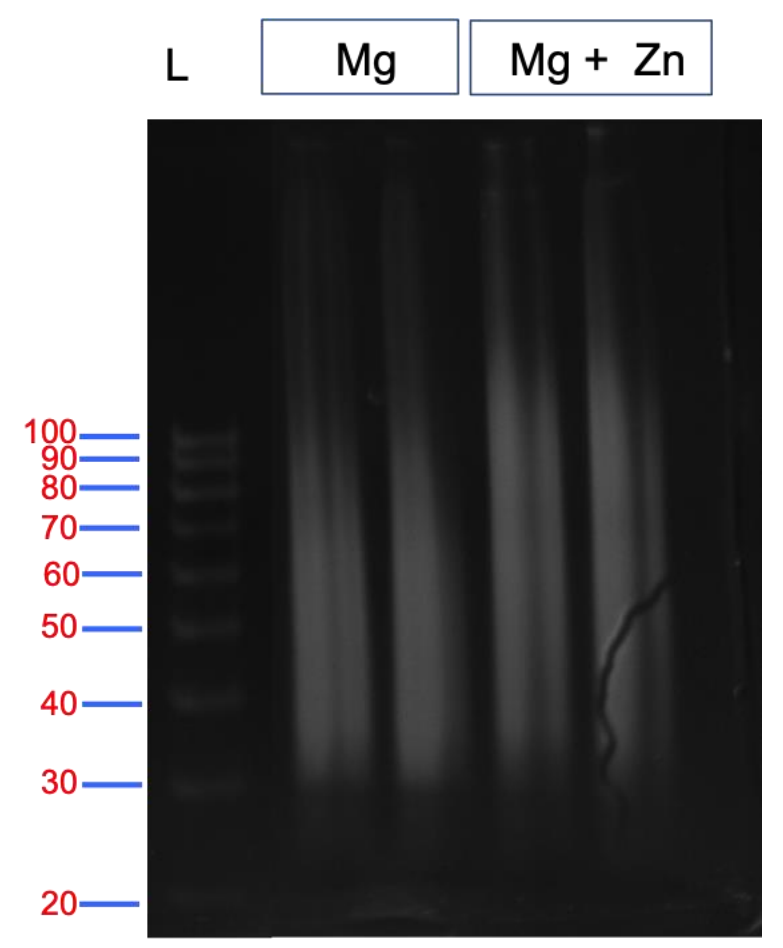

Figure S4: Length distribution of extensions upon addition of $\mathrm{Zn}^{2+}$ as seen on ssDNA gel

Extension reactions were run as mentioned in Materials and Methods section. Two biological replicates per test condition were then loaded onto a ssDNA gel $\left(\mathrm{Mg}^{2+}\right.$ on left and $\mathrm{Mg}^{2+}+\mathrm{Zn}^{2+}$ on right). Addition of $\mathrm{Zn}^{2+}$ increases the overall lengths of the extensions. 


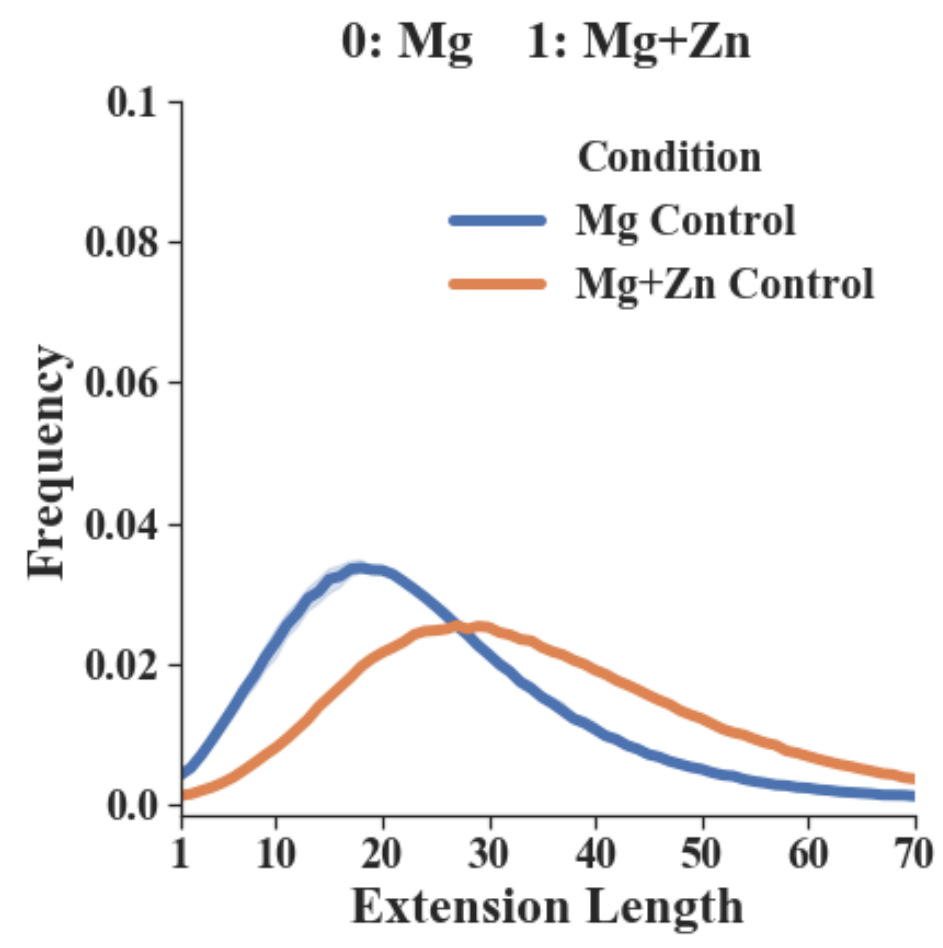

Figure S5: Length distribution of extensions upon addition of $\mathrm{Zn}^{2+}$ based on NGS data We calculated the mean frequency distribution of extension lengths for each condition (three biological replicates for each condition). Addition of $\mathrm{Zn}^{2+}$ caused a shift in probability distribution toward longer lengths. 


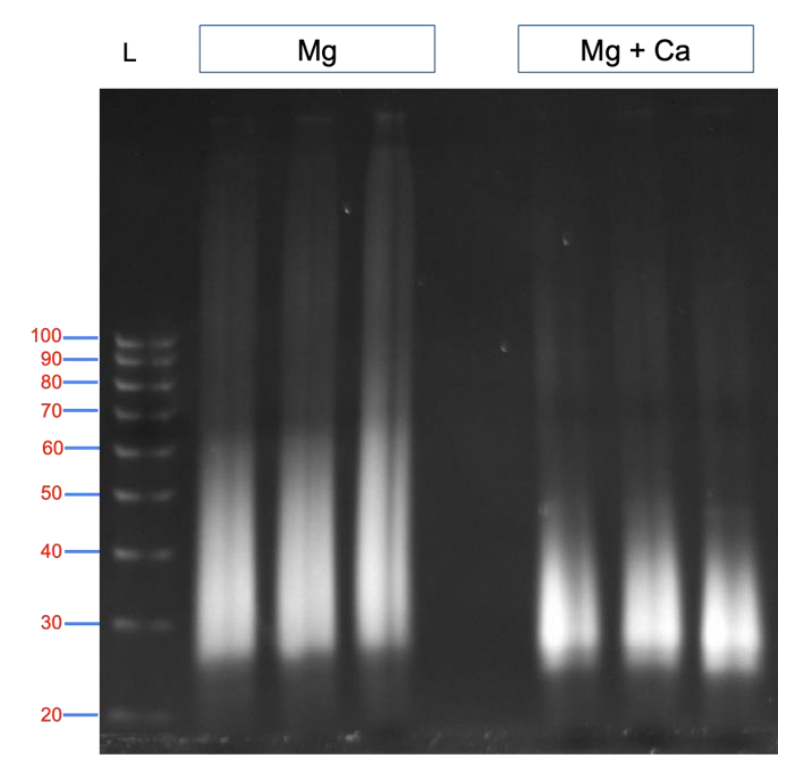

Figure S6: Length distribution of extensions upon addition of $\mathrm{Ca}^{2+}$ as seen on ssDNA gel

Extension reactions were run as described in Materials and Methods section. Three biological replicates per test condition were then loaded on a ssDNA gel $\left(\mathrm{Mg}^{2+}\right.$ on left and $\mathrm{Mg}^{2+}+\mathrm{Ca}^{2+}$ on right). Addition of $\mathrm{Ca}^{2+}$ decreases the overall lengths of the extensions. 


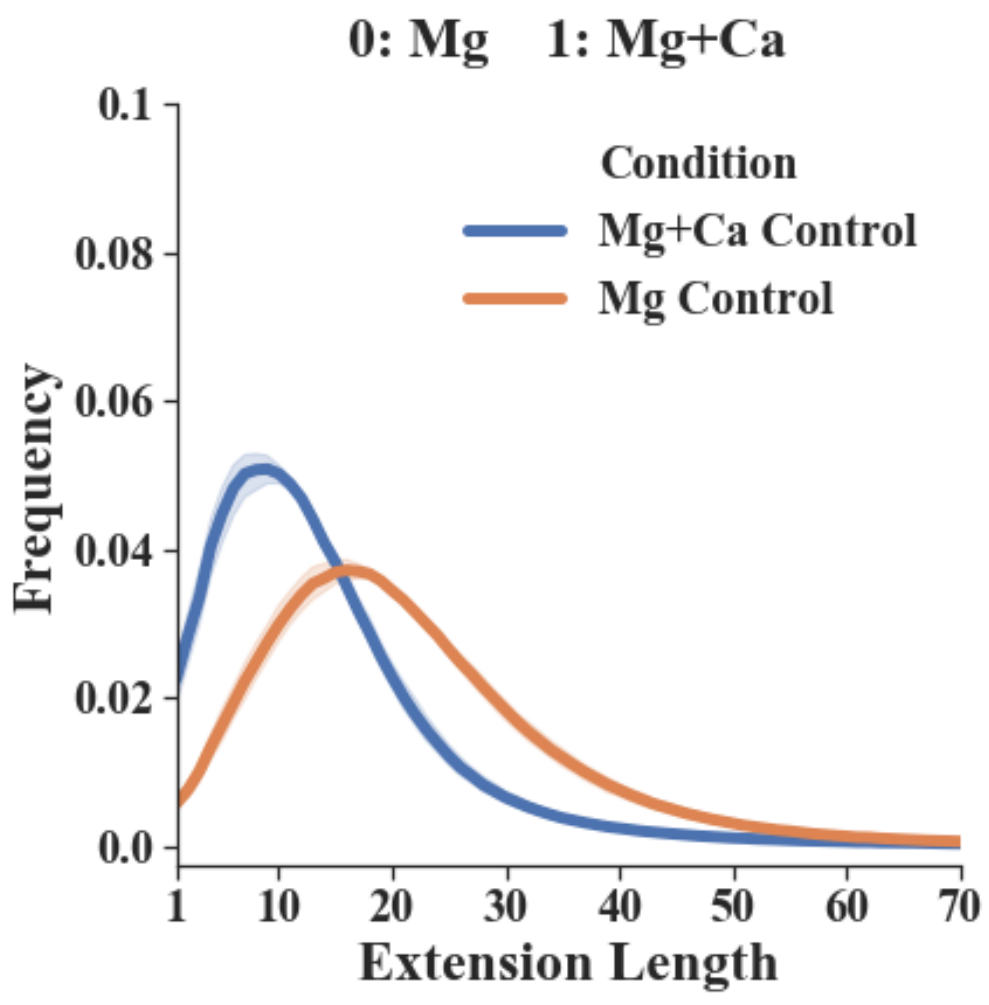

Figure S7: Length distribution of extensions upon addition of $\mathrm{Ca}^{2+}$ based on NGS data.

We calculated the mean frequency distribution of extension lengths for each condition (three biological replicates for each condition). Addition of $\mathrm{Ca}^{2+}$ caused a shift toward shorter lengths. 


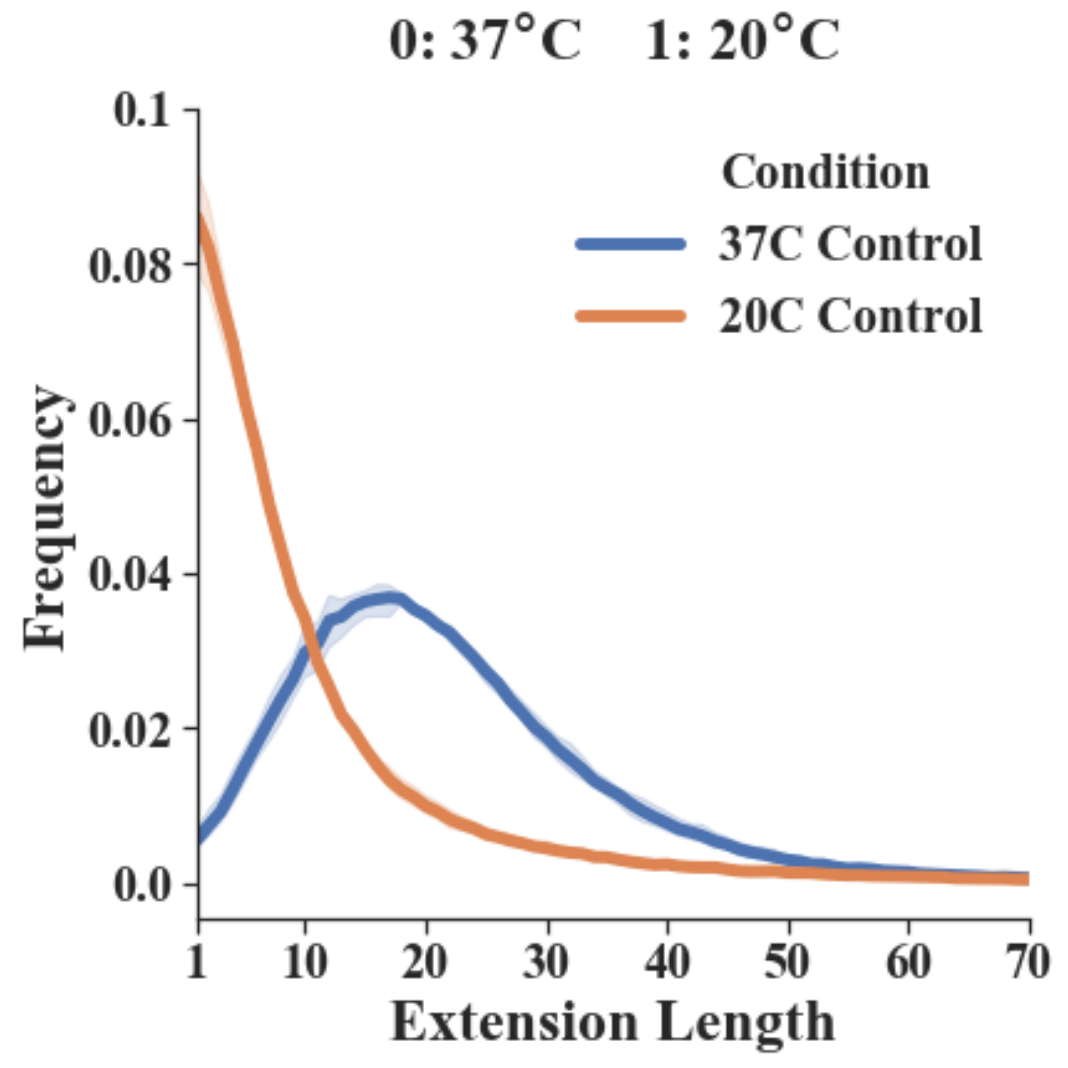

Figure S8: Length distribution of extensions upon using temperature as a signal based on NGS data We calculated the mean frequency distribution of extension lengths for each condition (three biological replicates for each condition). Reducing the temperature of the extension reaction to $20^{\circ} \mathrm{C}$ caused a shift toward shorter lengths. 

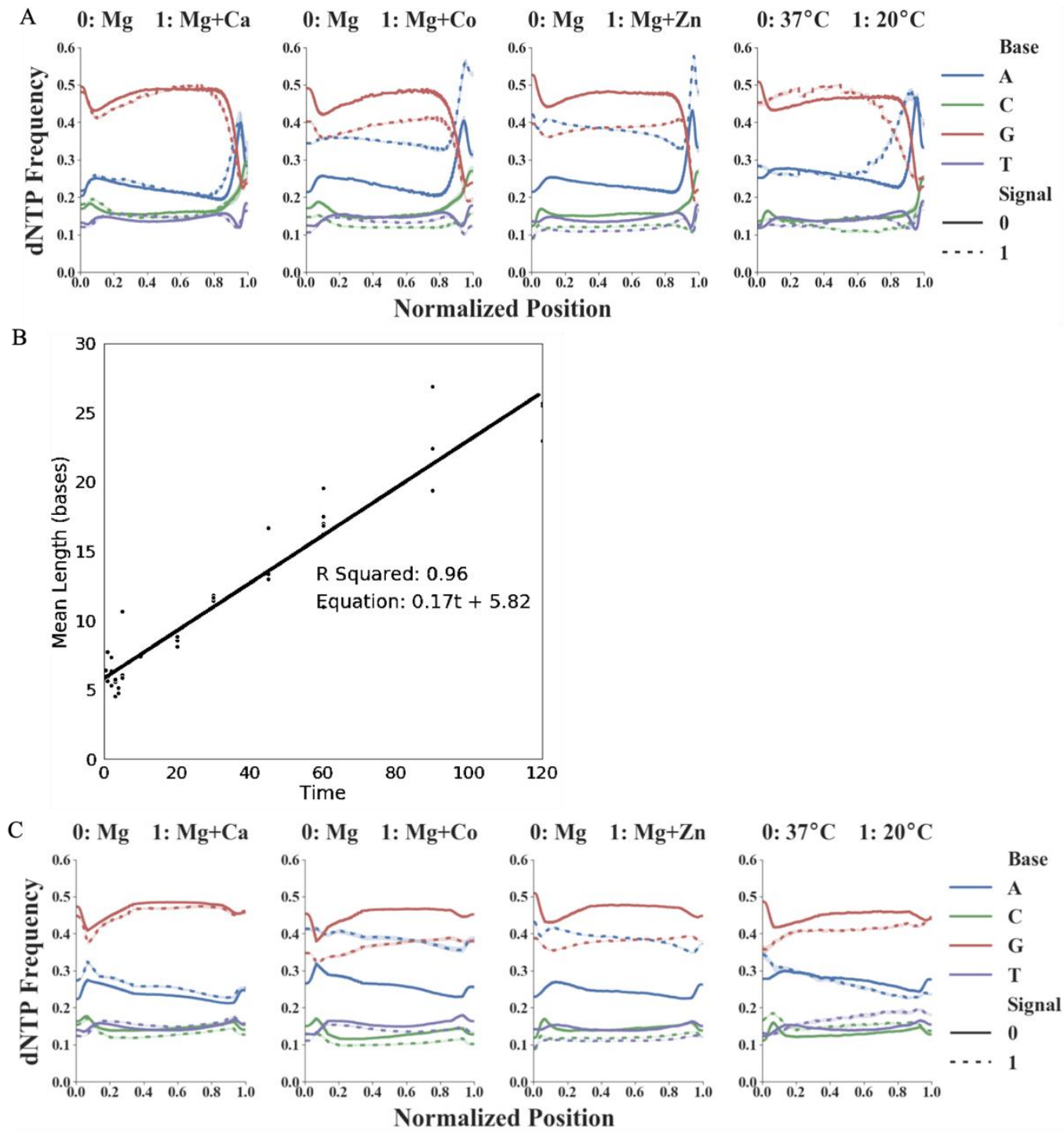

Figure S9: Anomalous dNTP composition initially found at the end of reads and rate of reaction measured for extensions with only $\mathbf{M g}^{2+}$ present

We observed a significant change in the individual dNTP frequency towards the ends of the ssDNA sequences synthesized. (A) Presents the significant change observed near the end of all reads with all the signals tested. Since we directly use $2 \mu \mathrm{L}$ of extension reaction for ligation, the diluted TdT seems to be adding dNTPs to the ssDNA after the recording experiment, during the 16-hour ligation step. (B) To prove that these dNTPs were not added during the extension reaction (i.e. after the reaction), we sampled extension reactions (with $\mathrm{Mg}^{2+}$ only) at several time points (Supplementary Text). We then calculated the mean extension length at each timepoint and applied a linear regression. The $\mathrm{R}^{2}$ value of 0.96 for a straight line indicates that our assumption of constant rate (assuming input signal does not change) is valid. The slope of 0.17 reveals an average incorporation rate of $0.17 \mathrm{dNTPs} /$ minute for this condition. Most importantly, the intercept of 5.82 indicates addition of 5.82 dNTPs (on average) either before or after the extension reaction. These are almost certainly being added after the extension reaction during the ligation step, which we conclude based on the anomalous behavior we see at the end of sequences in Panel A. (C) We created plots of the data from Panel A after trimming off last few dNTPs. See Materials and Methods for details on how these 5.8 bases were trimmed from the end of all sequences before further analysis. 


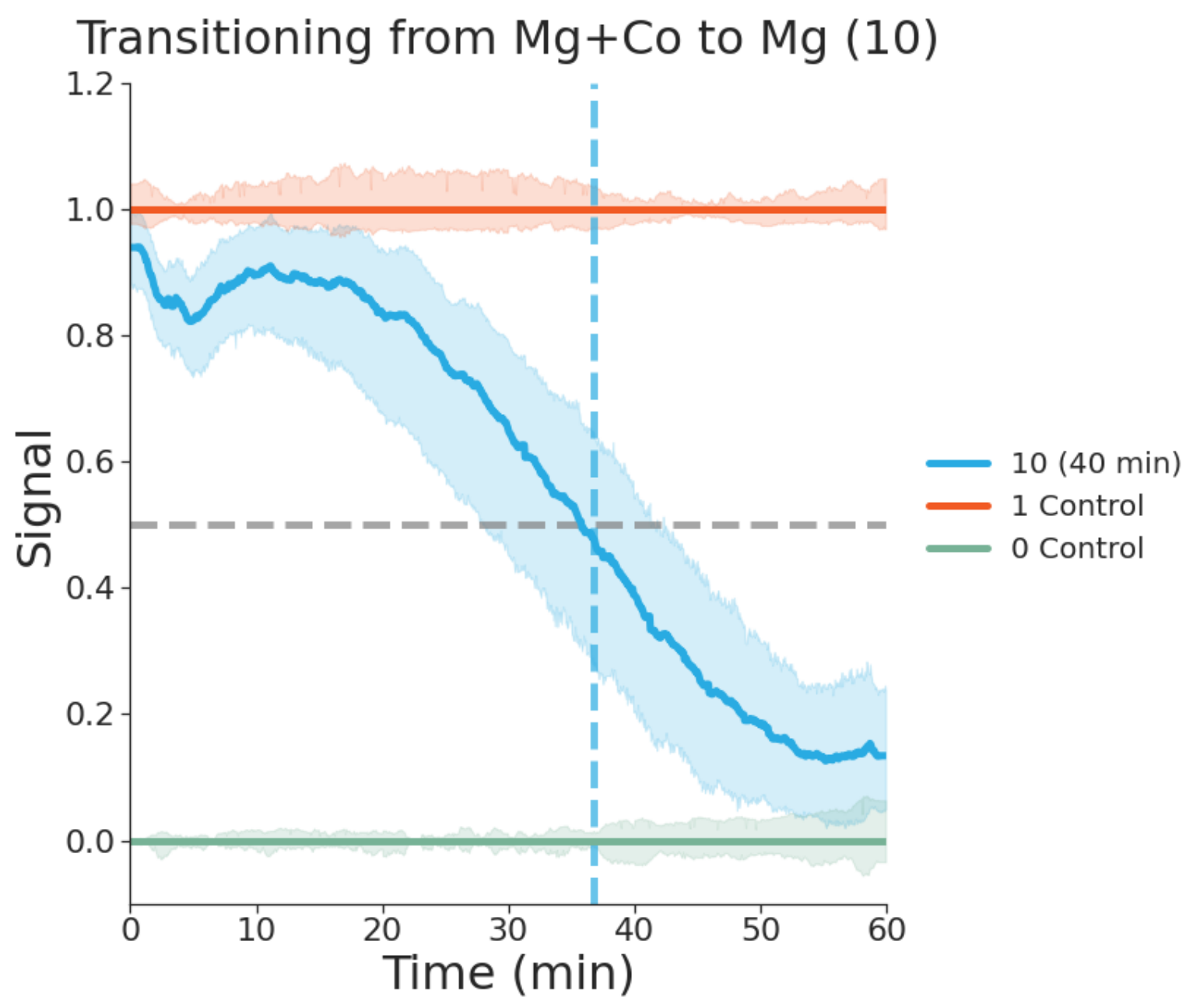

Figure S10: Recording a single $1 \rightarrow 0$ step change in $\mathrm{Co}^{2+}$ concentration onto ssDNA in vitro. $0.25 \mathrm{mM} \mathrm{Co}^{2+}$ was removed at 40 minutes to generate a $1 \rightarrow 0$ transition. This mean output signal across 6 biological replicates shows there is a difference in the preference of dNTP incorporated by TdT in the $\mathrm{Mg}^{2+}$ (green) and $\mathrm{Mg}^{2+}+\mathrm{Co}^{2+}$ (orange) control conditions. The plot further shows the changes from $1 \rightarrow 0$ for $\mathrm{Co}^{2+}$ removed at 40 minutes (blue). We were able to get a switch time of 36.7 minutes with a std. dev. of 9.9 minutes using methods used for $0 \rightarrow 1$ switch time predictions. We suspect the possible reason for higher variance in time prediction for this set-up was due to the ssDNA wash step at 40 minutes as discussed in Fig. S11. 

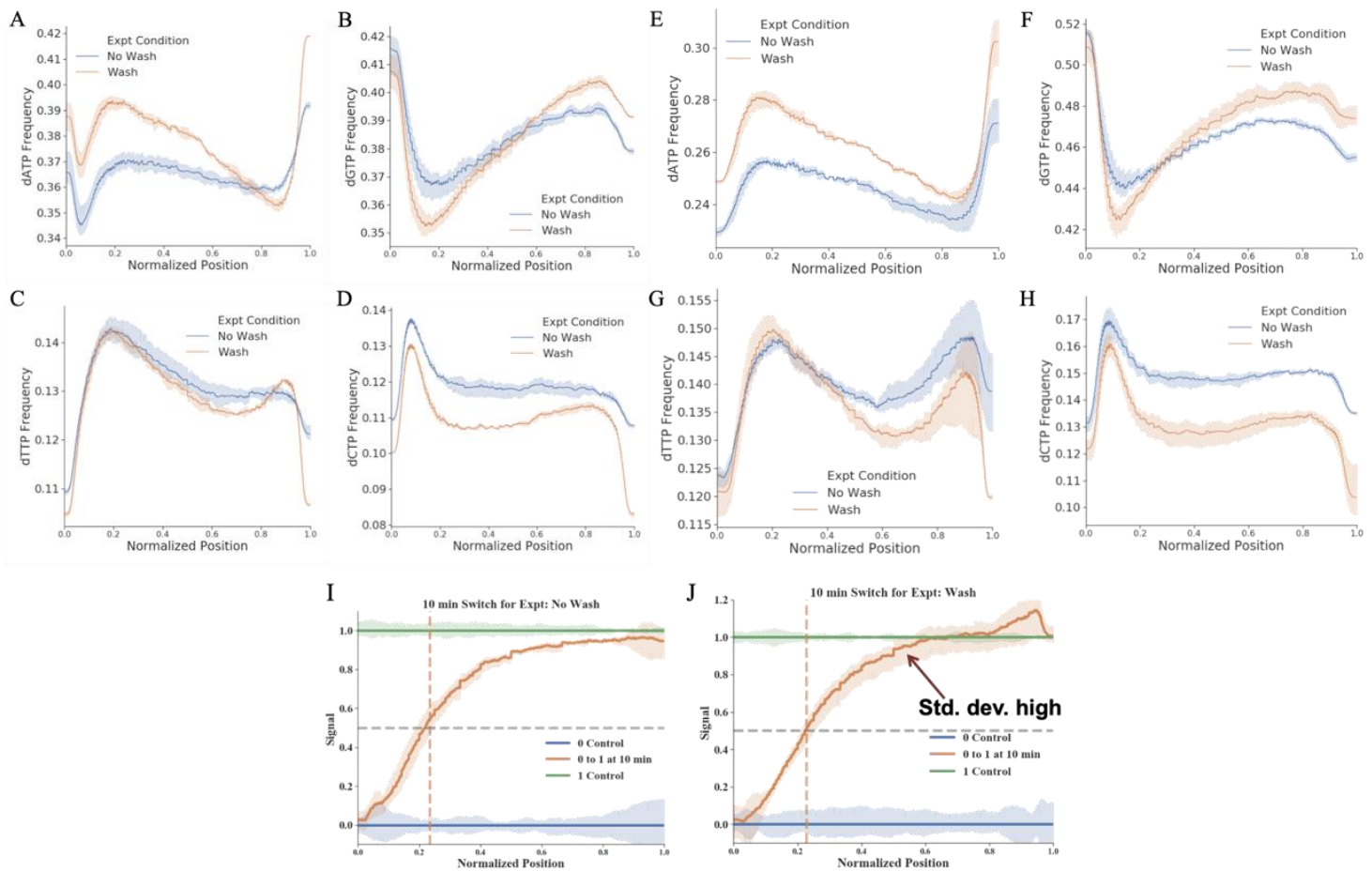

Figure S11: dNTP bias \& variability introduced by ssDNA wash columns

We present here a comparison of the composition of sequences retained when the extension reactions were directly used for ligation ("No Wash") vs. when the same extensions were put through a ssDNA wash kit ("Wash"). (A), (B), (C) and (D) show individual plots of each nucleotide frequency seen in extension reactions between No Wash vs Wash conditions for just $\mathrm{Mg}^{2+}$ extensions. $(E),(F),(G)$ and $(H)$ show individual plots of each nucleotide frequency seen in extension reactions between No Wash vs Wash conditions for $\mathrm{Mg}^{2+}+\mathrm{Co}^{2+}$ extensions. We observed a bias in overall dNTP content introduced by the columns used for ssDNA clean-up, when the reactions were washed after the recording experiment was stopped. ssDNA sequences with certain dNTP compositions were preferentially retained on the columns. (I) and $(\mathrm{J})$ are plots for time prediction for No Wash and Wash condition respectively. We carried out an input signal of $\mathrm{Co}^{2+} 0 \rightarrow 1$ at 10 minutes for a 1-hour extension. We obtained a time prediction of 12.8 minutes with $1.8 \mathrm{~min}$ std. dev. for No Wash condition. We obtained a time prediction of $12.4 \mathrm{~min}$ with a std. dev. of $1.2 \mathrm{~min}$ for the Wash condition. While the time predictions were very similar, there is a clear increase in variability (std. dev.) for the later part of the output signal recorded in (J) as compared to (I) (shown with a red arrow). Taken together, such biases and variability when introduced during the wash step for $0 \rightarrow 1 \rightarrow 0$ experiment at 40 minutes for replacing $+\mathrm{Co}^{2+}$ buffers with $-\mathrm{Co}^{2+}$ buffers (See Materials and Methods: Extension reactions for $0 \rightarrow 1 \rightarrow 0$ set-up) would cause more noise for the final 20 minutes of the recording. 


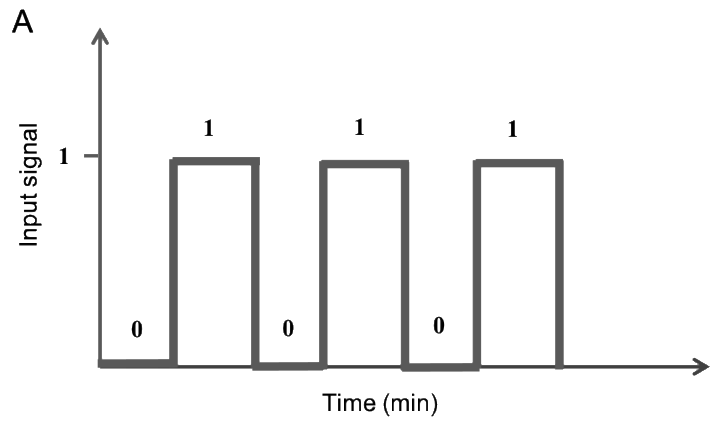

B

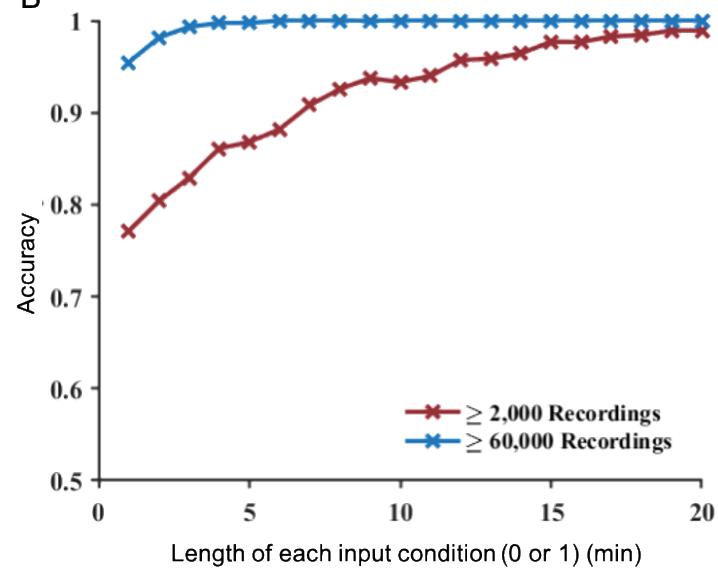

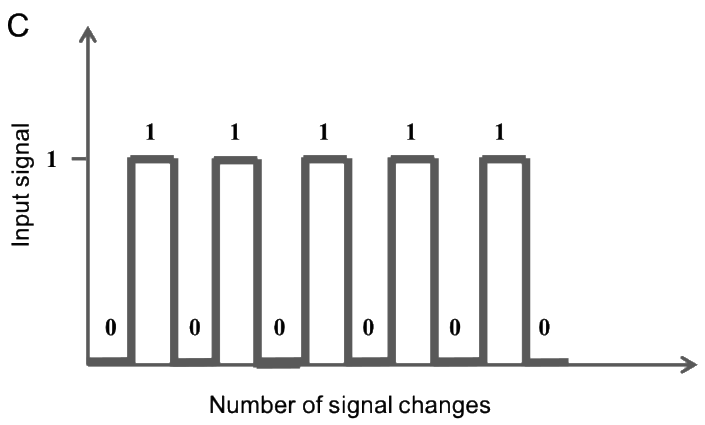

D

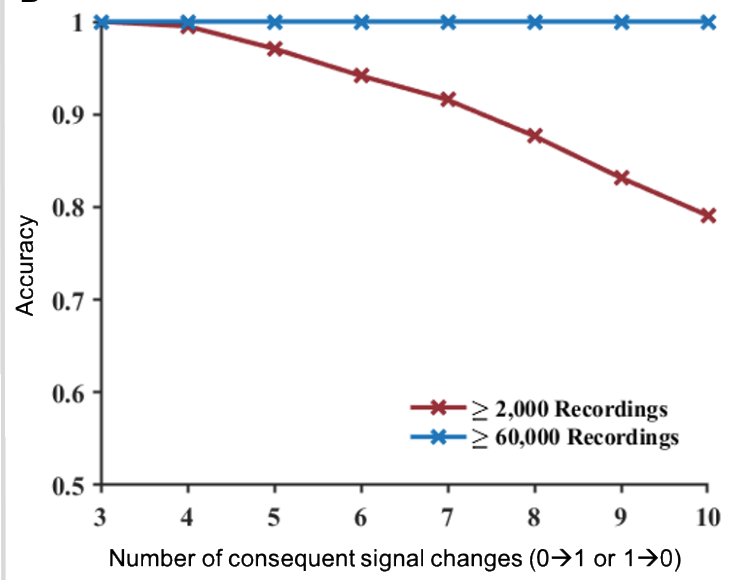

Figure S12: In silico characterization of shortest resolvable input signal changes and highest number of consecutive signal changes that could be resolved using TURTLES

In silico simulations based on experimental TdT parameters. For all simulations, we calculated the variability in two ways. The variability was either calculated across the first 100 nucleotides, in which there were at least 2000 recordings of all base numbers (red curve) or across the first 50 nucleotides, in which there were at least 60000 recordings of all base numbers (blue curve). (A) Representative signal for rapidly changing input from $0 \rightarrow 1$ or $1 \rightarrow 0$. (B) Output of in silico characterization of the signals represented by (A). We tried to find the shortest times of input changes from $0 \rightarrow 1$ or $1 \rightarrow 0$ that could be resolved using TURTLES. Simulations were carried out where the number of input signal changes were kept constant at 6 (e.g., $0 \rightarrow 1 \rightarrow 0 \rightarrow 1 \rightarrow 0 \rightarrow 1 \rightarrow 0$ ) while the length of time for each input condition ( 0 or 1 ) was varied. Even with just 2000 strands of ssDNA recorded (each $100 \mathrm{bp}$ in length), input signal changing every 1 minute was resolved with $>75 \%$ accuracy, and input signal changing every 10 minutes was resolved with $>90 \%$ accuracy. (C) Representative signal for multiple changes in input signal from $0 \rightarrow 1$ or $1 \rightarrow 0$. (D) Output of in silico characterization of the signals represented by $(\mathrm{C})$. Simulations were carried out with the duration of each input condition ( 0 or 1 ) kept constant at 10 minutes while varying the number of input signal changes. The accuracy of resolving this output signal was calculated across simulations. Even with just 2000 strands of ssDNA recorded (each 100bp in length), 3 input signal changes could be resolved with almost 100\% accuracy and 10 input signal changes of 10 minutes each could be resolved at about $80 \%$ accuracy. 

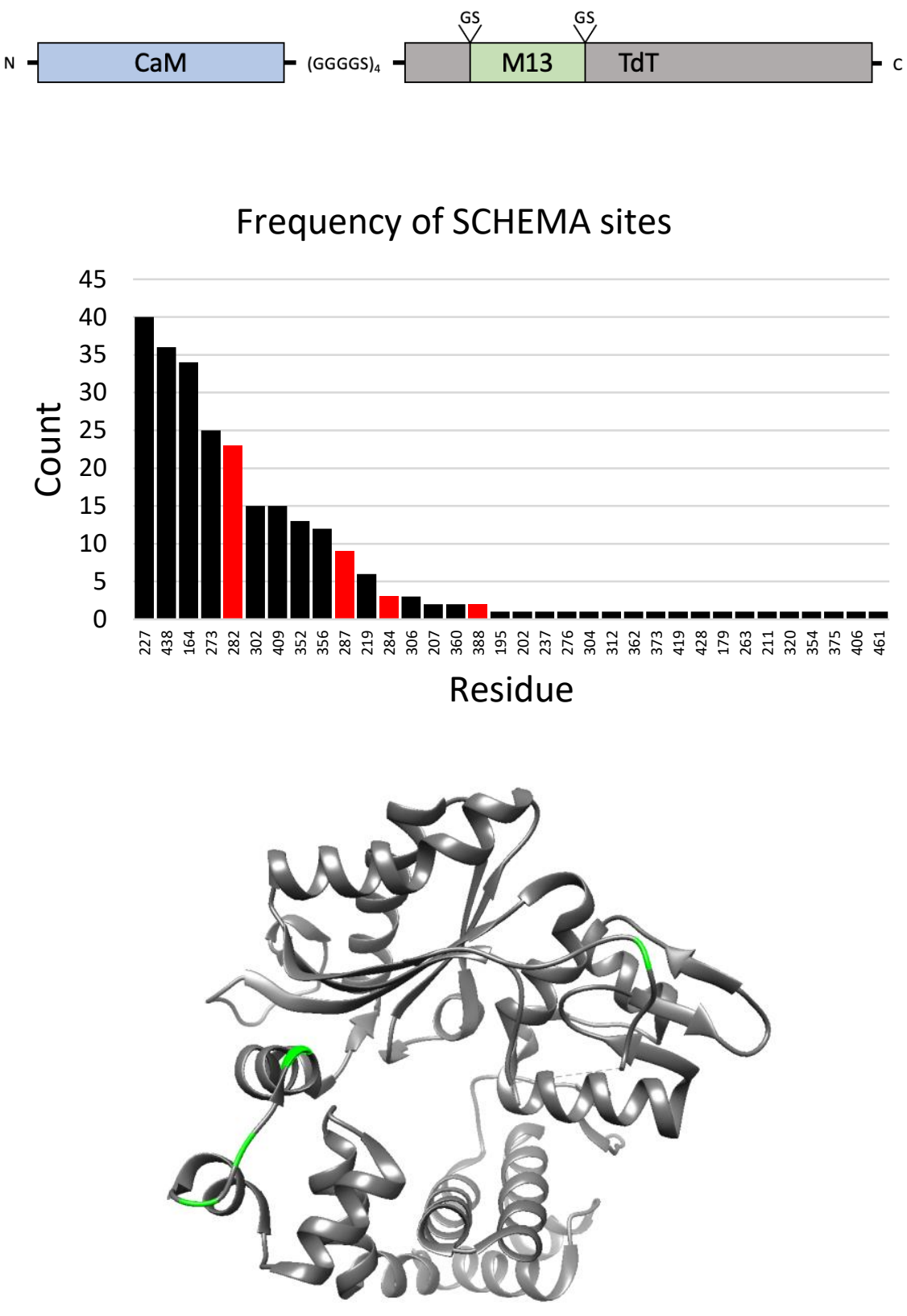

Figure S13: CaM-TdT(M13) design and SCHEMA crossover site frequency and locations. (A) schematic representation of CaM-TdT(M13) variants. (B) the number of times each crossover site appeared in the SCHEMA/RASPP analysis. Sites selected for fusions are colored in red. (C) ribbon diagram of the catalytic core of the short isoform of murine TdT (PDB ID: 4i27) with fusion site residues colored in green. Fusion sites were selected to minimize steric interference and target polymerase regions required for DNA binding $(282,284,287)$ or catalysis $(388)$. 


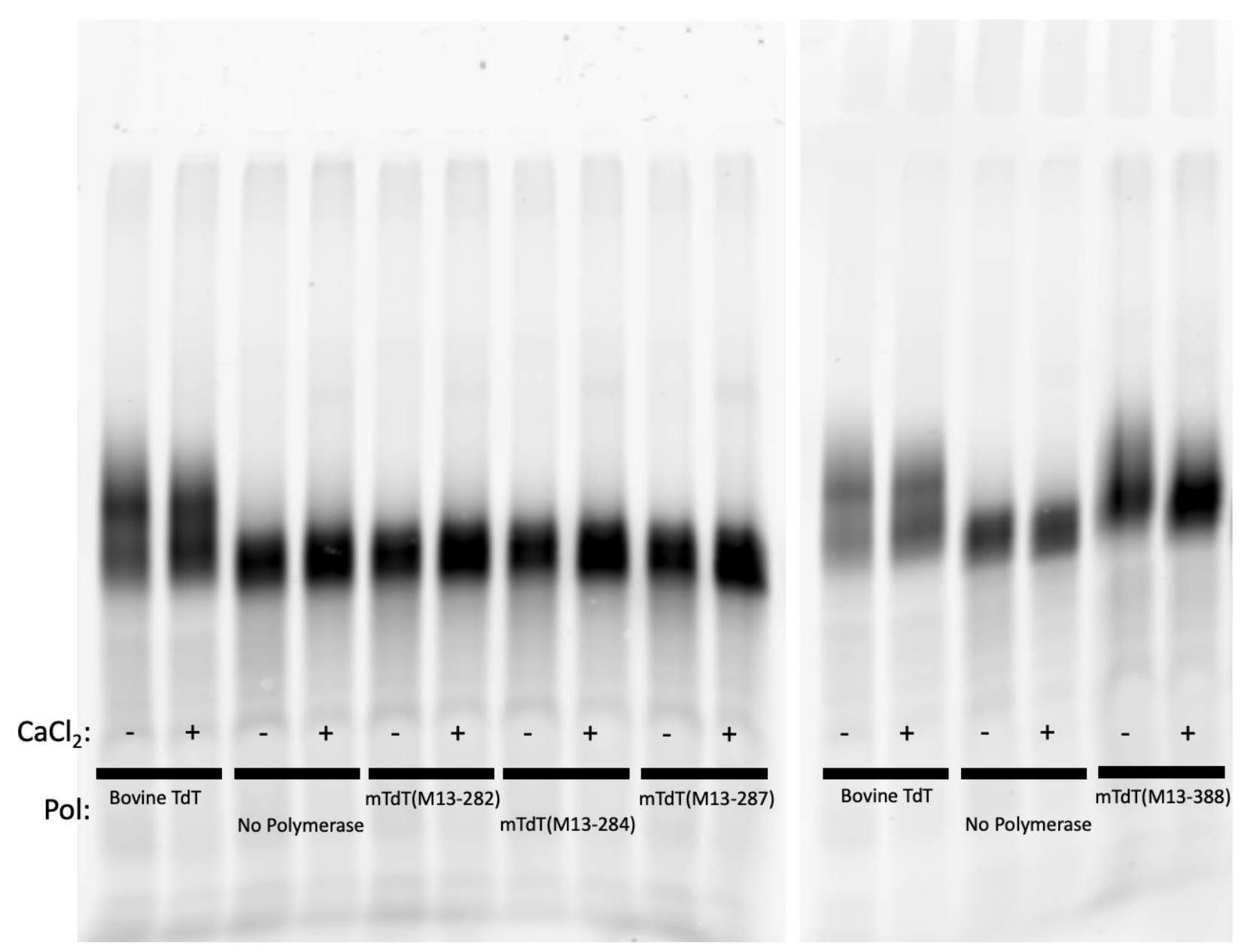

Figure S14: Primer extension activity of M13 TdT fusion proteins. Fusion proteins were screened by assaying the activity of the M13 fusion variants without N-terminal CaM to ensure that $\mathrm{mTdT}$ remained active with the M13 fusion. Fusions were expressed in PURExpress and their activity was screened by primer extension. Primer extension reactions were performed without added $\mathrm{CaCl}_{2}$ (indicated by "-") and with $1 \mathrm{mM} \mathrm{CaCl}$ (indicated by "+"). The reaction products were visualized by urea-PAGE (see methods). Fusions are labelled as $\mathrm{mTdT}(\mathrm{M} 13-\# \# \#)$, where the number indicates the residue immediately preceding the GS-M13-GS fusion. 


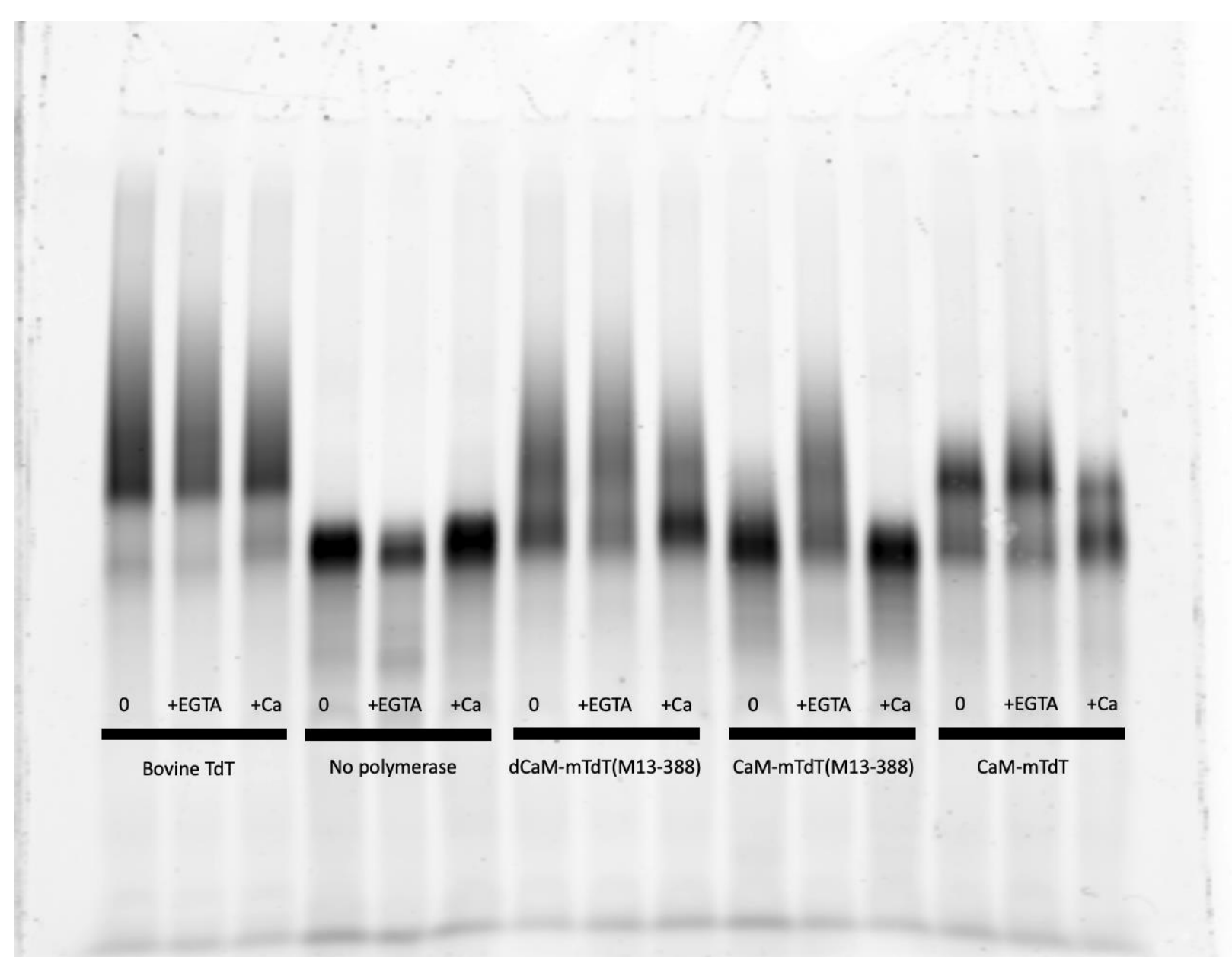

Figure S15: Primer extension activity of CaM-mTdT(M13-388), dCaM-mTdT(M13-388), and mTdT(M13-388). The activity of each polymerase was tested in three conditions where "0" indicates reactions with no added calcium or EGTA, "+EGTA" indicates reactions supplemented with $1 \mathrm{mM}$ EGTA, and "+Ca" indicates reactions supplemented with $1 \mathrm{mM} \mathrm{CaCl}$. dCaM-mTdT(M13-388) contains CaM mutations D20A, D56A, D93A, and D129A that ablate the calcium-binding capability of CaM(49). CaMmTdT consists only of mTdT with N-terminal CaM fusion. 


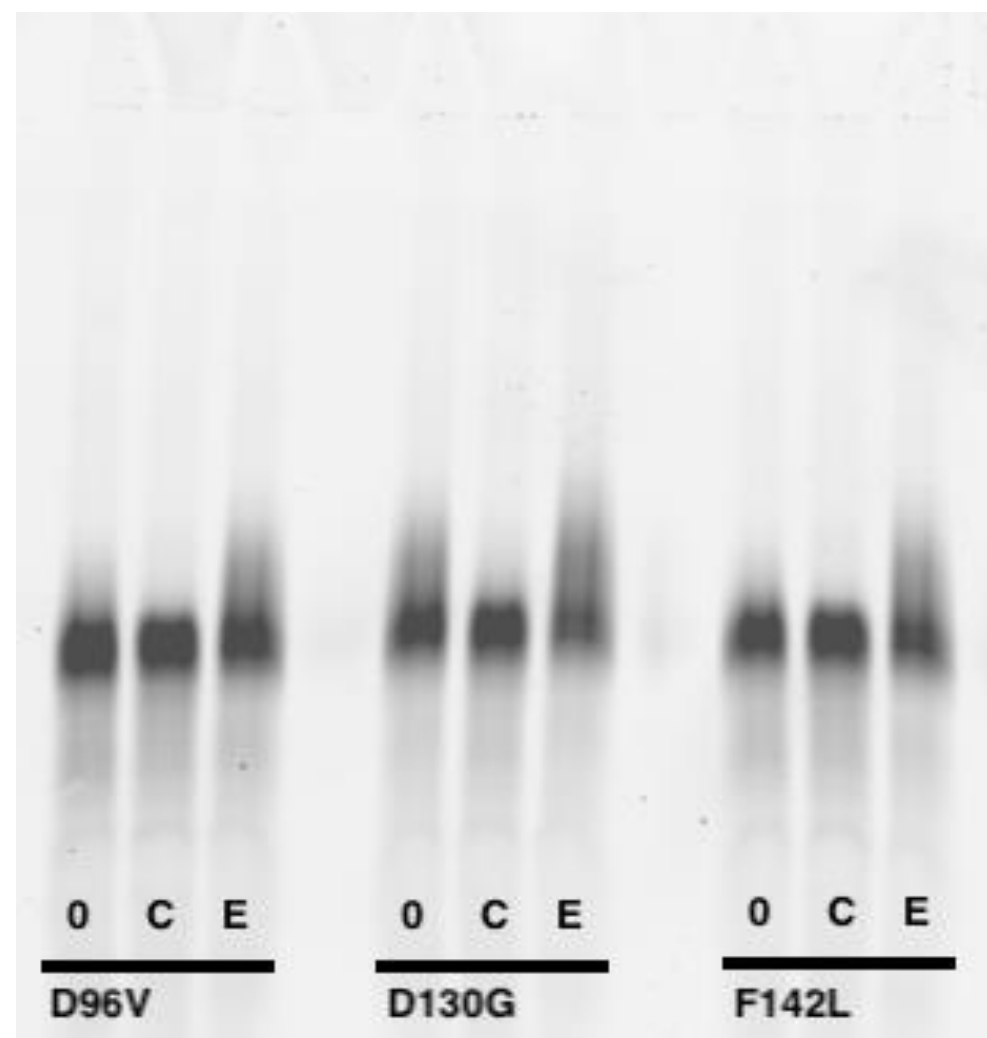

Figure S16: Primer extension activity of CaM affinity variants. the activity of calcium affinity variants was tested by primer extension and visualized by urea PAGE in non-supplemented ("0"), $1 \mathrm{mM} \mathrm{CaCl} 2$ added ("C"), and 1mM EGTA added conditions ("E"). Crotti et al. report effective calcium affinities of $38 \mu \mathrm{M}, 150$ $\mu \mathrm{M}$, and $15 \mu \mathrm{M}$ respectively as compared to $2.8 \mu \mathrm{M}$ for wildtype CaM. 


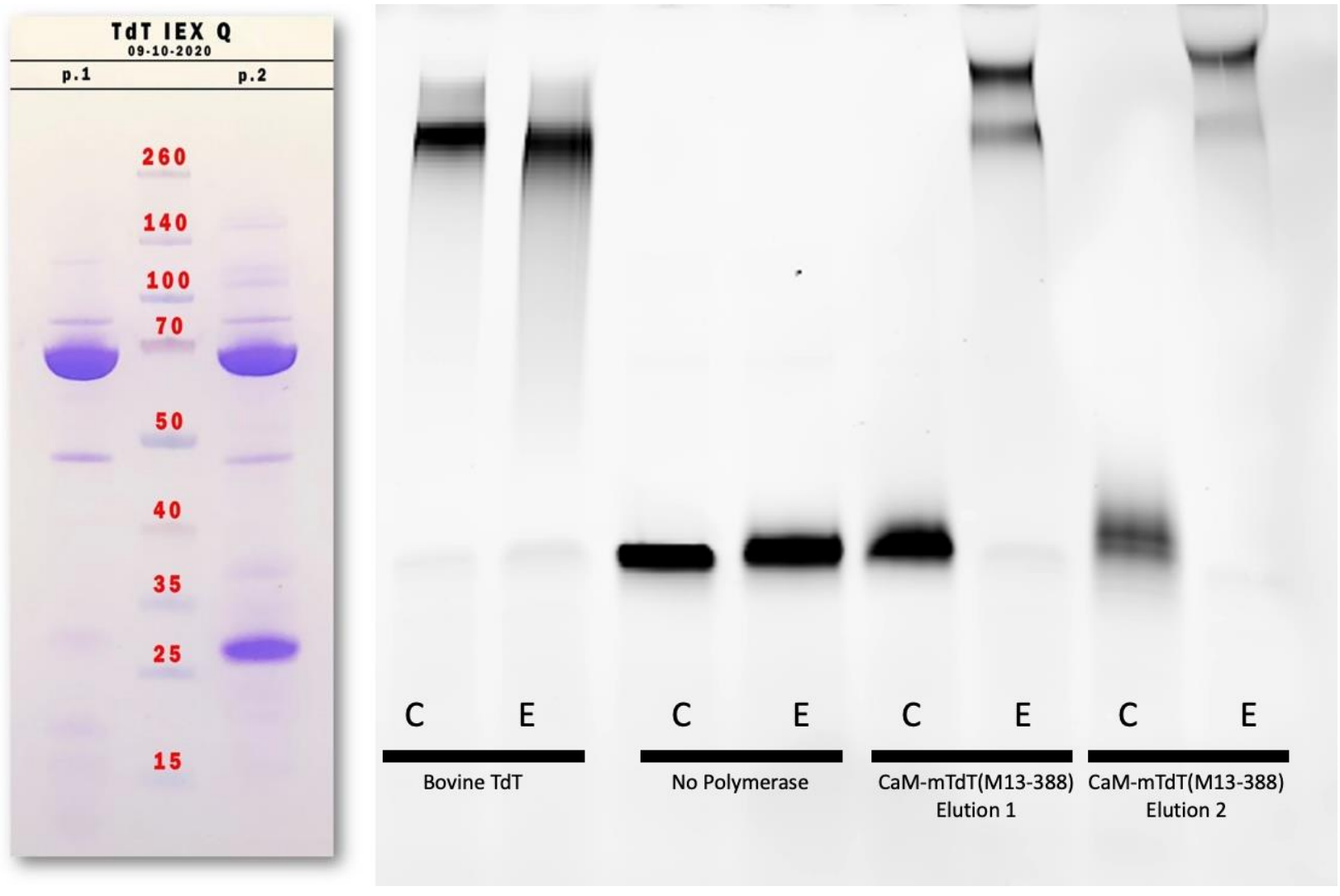

Figure S17: Primer extension activity of purified CaM-mTdT(M13-388). A) Anion exchange elutions 1 (p.1) and 2 (p.2), visualized by PAGE. CaM-mTdT(M13-388) appears at approximately 70kDa. (B) Following buffer exchange, the activity of both fractions was assayed by primer extension and compared to the wildtype polymerase and MBP-CaM-mTdT(M13-388) (from PURExpress). $1 \mathrm{mM} \mathrm{CaCl} 2$ added ("C"), and $1 \mathrm{mM}$ EGTA added conditions ("E"). 

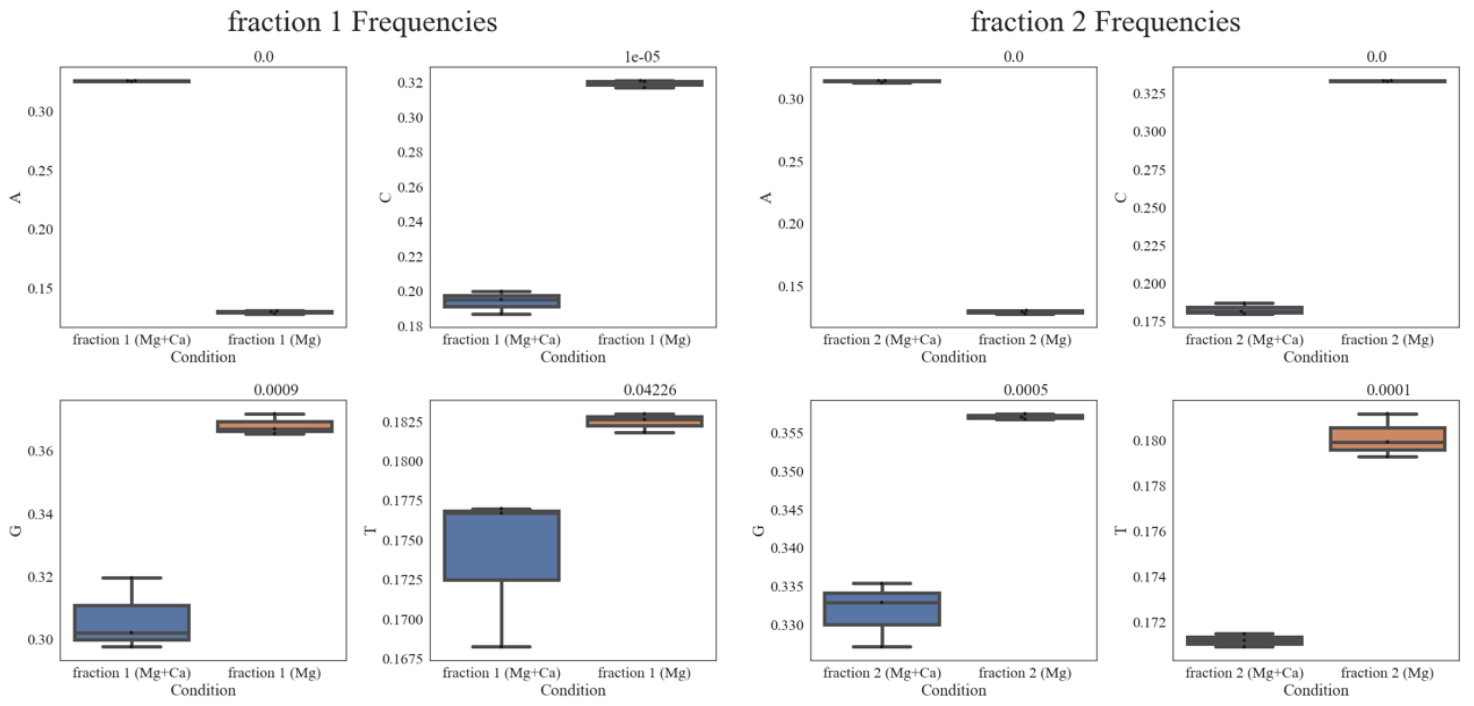

Figure S18. Comparison of purified CaM-mTdT(M13-388) fractions. base incorporation frequencies of $\mathrm{CaM}-\mathrm{mTdT}(\mathrm{m} 13-388)$ elution fractions 1 and 2 in primer extension reactions. 

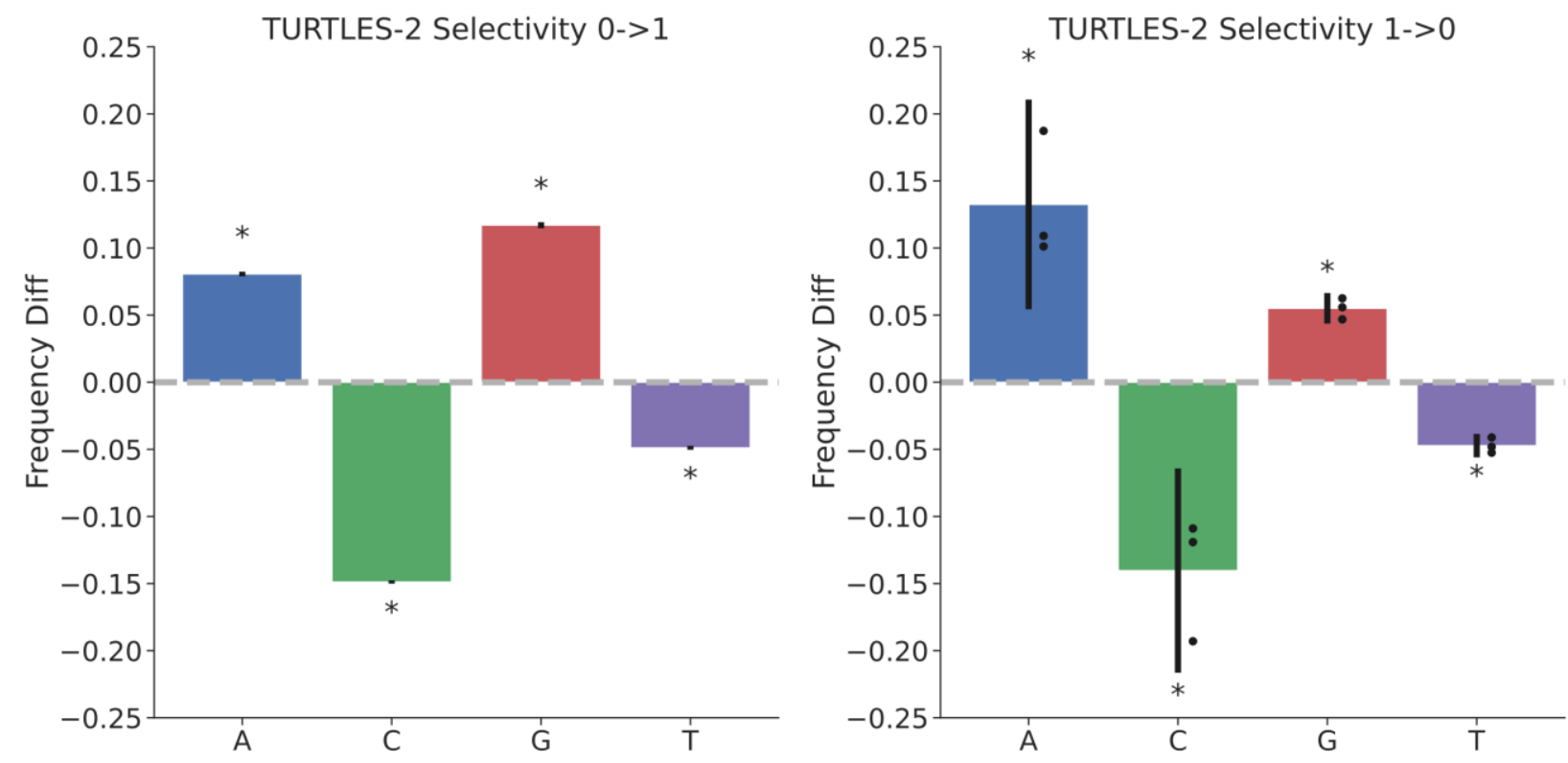

Figure S19: Incorporation preference of two-polymerase recording system. The difference in base selectivity of TURTLES-2 in the presence of calcium as compared to calcium-free conditions. Individual points (black dots) are added to the right plot to highlight the variability between each replicate's 1 frequency and the mean 0 frequency of each base. 

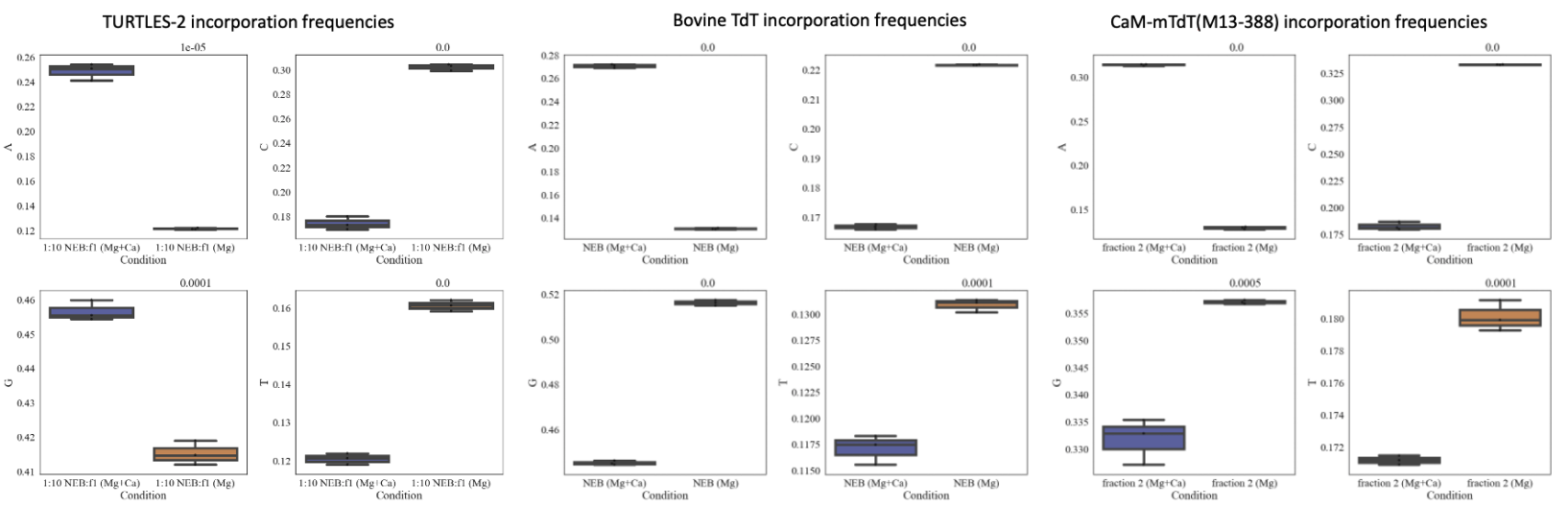

Figure S20: TURTLE-2 Selectivity comparison. Incorporation frequencies of each base for the twopolymerase TURTLES-2 system, bovine TdT, and purified CaM-mTdT(M13-388). 


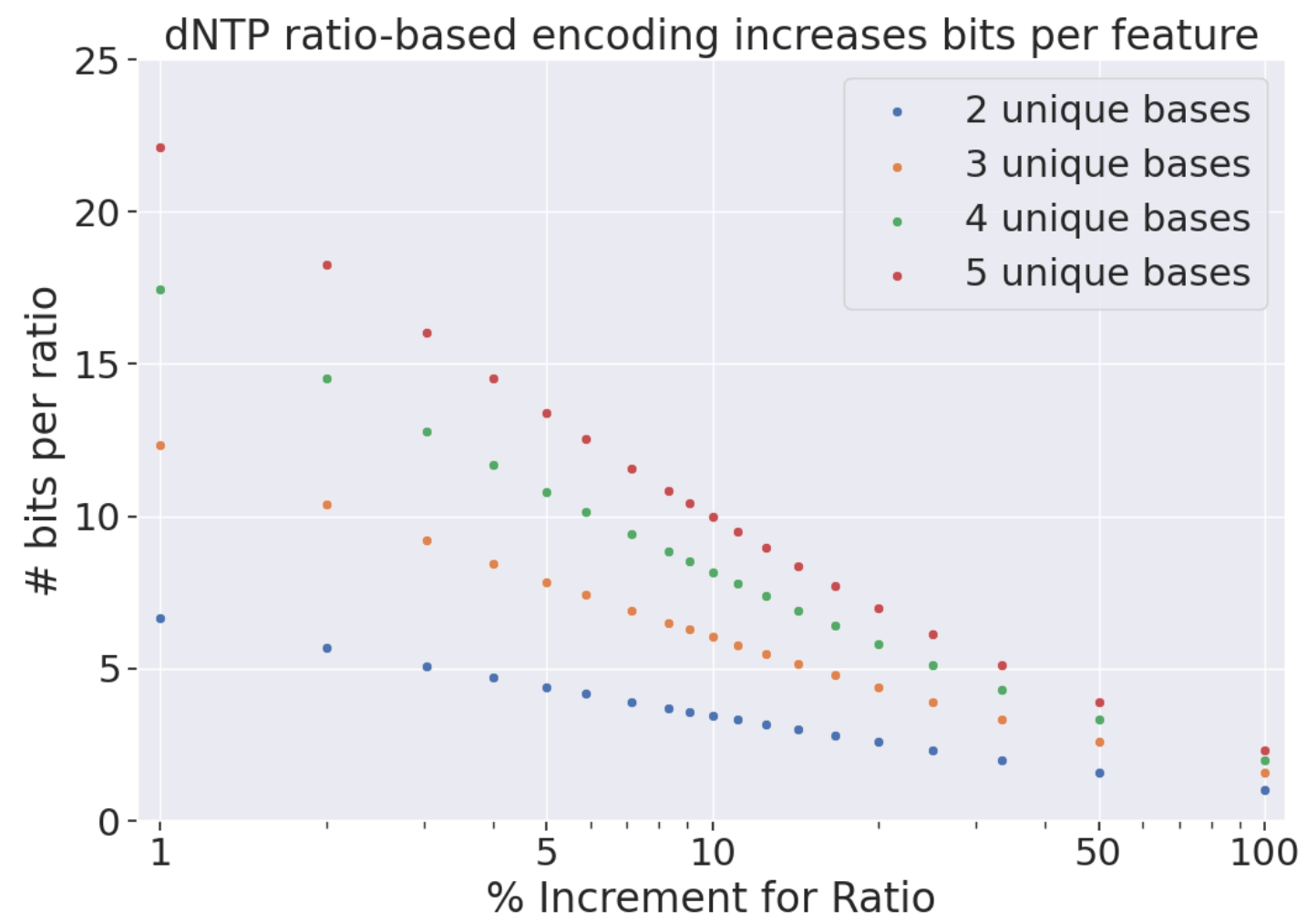

Figure S21: Digital data storage capacity of a TURTLES-based system that employs changes in concentration of nucleotides supplied over time. The data density of recordings is determined by the number of possible input concentrations of dNTPs (ratios) that yield distinguishable outputs. For example, with 4 unique bases (A, C, G, and T) and a set of ratios built in increments of $5 \%$ (e.g. $35 \% \mathrm{~A}, 20 \% \mathrm{C}, 15 \%$ $\mathrm{G}, 30 \% \mathrm{~T}$ ), the maximum amount of data stored per ratio is $11 \mathrm{bits}$, as there are $2^{11}$ possible compositions of $A, C, G$, and $T$ that add to $100 \%$ and use $5 \%$ increments for each nucleotide. Even greater densities are possible with smaller increments or use of unnatural bases. The main challenge of this system would be inferring back out the ratio at each timepoint from a population of sequences, especially at fine-grained ratio increments (e.g. at $5 \%$ increments). Some potential strategies to address these issues include reserving a base to act as a buffer between signals, increasing the length of each encoded signal, and using error correcting codes. 



\section{Supplementary Tables}

\begin{tabular}{|c|c|c|}
\hline Name & Sequence & Notes \\
\hline CS1 & ACACTGACGACATGGTTCTACA & \\
\hline CS1_5N & ACACTGACGACATGGTTCTACANNNNN & $\begin{array}{l}\text { Degenerate "N " } \\
\text { bases are } \\
\text { distributed } 25 \% \\
\mathrm{~A}, 15 \% \mathrm{C} \\
45 \% \mathrm{G}, 15 \% \mathrm{~T}\end{array}$ \\
\hline $\begin{array}{l}\text { FAM_N } \\
\text { B }\end{array}$ & $\begin{array}{l}\left(6{ }^{\prime}-\right. \\
\text { FAM)A*}{ }^{*} C^{*} A^{*} C^{\star} \text { TGACGACATGGTTCTACAACCGGTATGTACGGCGGTCGGTTAT } \\
\text { CGTA }\end{array}$ & $\begin{array}{l}\text { "*" follows } \\
\text { phosphorothioate } \\
\text { d bases }\end{array}$ \\
\hline $\begin{array}{l}\text { AMD00 } \\
6\end{array}$ & AGGCTAGTCGTCTGTATAGG & \\
\hline P1 & CTCCAGAGGCCACAGGGTCTGCTGACCAATTAACCGAAGAAC & \\
\hline P2 & TGTTCCTCGTCCTCGCTGCCCTTGGCTGTCATCATCTGC & \\
\hline P3 & AGGCCACAGAGGACGGGTCTGCTGACCAATTAACCGAAGAAC & \\
\hline P4 & AACTGTTGTTCCTCGCTGCCCTTGGCTGTCATCATCTGC & \\
\hline P5 & CCTTCGAGAAGTTCGGGTCTGCTGACCAATTAACCGAAGAAC & \\
\hline P6 & CGGCTTGGCTGTTTGCTGCCCTTGGCTGTCATCATCTGC & \\
\hline P7 & AAGGTAAAGGCTGGGGGTCTGCTGACCAATTAACCGAAGAAC & \\
\hline P8 & ACACGGATAGCTTTGCTGCCCTTGGCTGTCATCATCTGC & \\
\hline P9 & AATTCGACGACGACGACAAAGCTGACCAATTAACCGAAGAACAAATC & \\
\hline P10 & TGCGAAATCTTTTTTACCGCGGAACCTCCCCCTCCAGAAC & \\
\hline P11 & TGATGACAGCCAAGGGCAGCGAGGACGAGGAACAACAGTTG & \\
\hline P12 & GTTAATTGGTCAGCAGACCCTGTGGCCTCTGGAGAAGTGATC & \\
\hline P13 & TGATGACAGCCAAGGGCAGCGAGGAACAACAGTTGTTGCACAAG & \\
\hline P14 & GTTAATTGGTCAGCAGACCCGTCCTCTGTGGCCTCTGGAG & \\
\hline P15 & TGATGACAGCCAAGGGCAGCAAACAGCCAAGCCGCAAAG & \\
\hline P16 & GTTAATTGGTCAGCAGACCCGAACTTCTCGAAGGTACTTTCTAAAATATCGC & \\
\hline P17 & TGATGACAGCCAAGGGCAGCAAAGCTATCCGTGTTGACCTTGTG & \\
\hline P18 & GTTAATTGGTCAGCAGACCCCCAGCCTTTACCTTCCTGCTG & \\
\hline P19 & GTTCTGGAGGGGGAGGTTCCGCGGTAAAAAAGATTTCGCAGTACG & \\
\hline P20 & TCAGCTTTGTCGTCGTCGTCGAATTCAGACCCGTCGACGATG & \\
\hline P21 & AGATCCAAAGTGACAAAAGCGGGTCTGCACGCCGTAAATG & \\
\hline P22 & TGCATTTGCGTGAAACGCAGGCTGCCGGAACTTAAACGCC & \\
\hline P23 & AAAGTGACAAAAGCCTGCGTGGGTCTGCACGCCGTAAATG & \\
\hline P24 & GCTTTTTGCATTTGCGTGAAGCTGCCGGAACTTAAACGCC & \\
\hline P25 & AAAGCCTGCGTTTCACGCAAGGGTCTGCACGCCGTAAATG & \\
\hline P26 & AAAAACCCAGCTTTTTTGCATGCTGCCGGAACTTAAACGCC & \\
\hline P27 & AAAGTACCTTCGAGAAGTTCGGGTCTGCACGCCGTAAATG & \\
\hline P28 & ACTTTGCGGCTTGGCTGTTTGCTGCCGGAACTTAAACGCC & \\
\hline P29 & AGATCCAAAGTGACAAAAGCGGGTCTGCACGCCGTAAATG & \\
\hline P30 & TGCATTTGCGTGAAACGCAGGCTGCCGGAACTTAAACGCC & \\
\hline P31 & AAAGTGACAAAAGCCTGCGTGGGTCTGCACGCCGTAAATG & \\
\hline P32 & GCTTTTTGCATTTGCGTGAAGCTGCCGGAACTTAAACGCC & \\
\hline P33 & AAAGCCTGCGTTTCACGCAAGGGTCTGCACGCCGTAAATG & \\
\hline P34 & AAAAACCCAGCTTTTTGCATGCTGCCGGAACTTAAACGCC & \\
\hline P35 & AAAGTACCTTCGAGAAGTTCGGGTCTGCACGCCGTAAATG & \\
\hline P36 & ACTTTGCGGCTTGGCTGTTTGCTGCCGGAACTTAAACGCC & \\
\hline
\end{tabular}




$\begin{array}{ll}\text { S1 } & \text { GCATTCTGGTATGCCGTCCG } \\ \text { S2 } & \text { GGTGGACGAAATGATCCGCG } \\ \text { S3 } & \text { GTTCGTGTGTCGCGTAGCGG } \\ \text { S4 } & \text { GGCTGAAGCGGTAAGTATGTTGG } \\ \text { S5 } & \text { CGAGGAGGAAATCCGCGAAGC } \\ \text { S6 } & \text { GATCACTTCTCCAGAGGCCACAG } \\ \text { S7 } & \text { GAGCCTTCCATTCCCTATTACCAG } \\ \text { S8 } & \text { GTCCGGCGTAGAGGATCGAG } \\ \text { S9 } & \text { CACTTGACAAGGAACTGAAGGCC }\end{array}$

Table S1: DNA substrate sequences and primer sequences 
MGHHHHHHHHHHSSGHIDDDDKHADQLTEEQIAEFKEAFSL FDKDGDGTITTRELGTVMRSL LQNPTEAELQDMINEVDADGNGTIDFPEELTMMARKMKDTDSEEETREAFRVFDKDGNGY I SAAELRHVMTNLGERLTDEEVDEMIREADID AEKWERMGFRTLSKIQSDKSLRFTQMQRAGELYYEDLVSCVNRPEAEAVSMLVKEAVVTFLPDALVTMTGGERRGKMTGHDVDHA PRTRVVELEAESEEEIFAHLGLDYIEPWERN

MGHнHннннНнHSSGH DDDDKHMMKIEEGKLVIWINGDKGYNGLAEVGKKFEKDTGI KVTVEHPDKLEEKF QQVAATGDG PDI IFWAHDRFGGYAQSGLLAEIT PDKAFQDKLYPFTWDAVRYNGKLIAYPIAVEALSLIYNNDLLLPNPPKTW EEI PALDKELKAKGKSALMFNLQEPYFTWPLIAADGGYAFKYENGKYDI KDVGVDNAGAKAGLTELVDLIKNKHMNADTDYSIAEAAFNKGETAMT I NGPWAWSNIDTSKVNYGVTVLPTFKGQPSKP FVGVLSAG INAAS PNKELAKEELEM

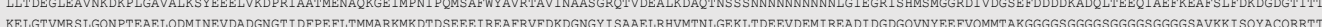
LNNYNOLFTDALDILAENDELRENEGSCLAFMRASSVLKSLPFPITSMKDTEGIPCLGDKVKSII EGIIEDGESSEAKAVLNDERYKSFKLFTSVFGVGLKTAEKWFRMGERTLSKIOSDKSGSARRKWOKTGHAVRAIGRLSSGSLRFTOMOK AGFLYYEDLVSCVNRPEAEAVSMLVKEAVVTFLPDALVTMTGGERRGKMTGHDVDFLITSPEATEDEEQOLLHKVTDFWKOOGLLLYCDILESTFEKFROPSRRVDALDHFQKCFLILKLDHGRVHSEKSGQQEGKGWKAIRVDLVMCPYDRRA FALLGWTGSRQFERDLRRYATHERKMMLDNHALYDRTKRVELEAESEEEIFAHLGLDYIEPWERNA

MGHнНннннНнHSSGHI DDDDKHMMKIEEGKLVIWINGDKGYNGLAEVGKKFEKDTGIKVTVEHPDKLEEKFPQVAATGDGPDII FWAHDRFGGYAQSGLLAEIT PDKAFQDKLYPFTWDAVRYNGKLIAYPIAVEALSLIYNKDLLPNP PKTW

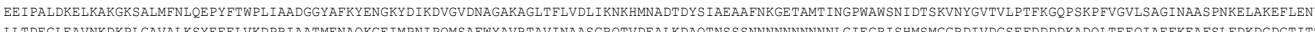

MBP-CaM-mTdT(M13-284) LHIDEGLEA LNMYNQLETDA AGFLYYEDLVSCVNRPEAEAVSMLVKEAVVTFLPDALVTMTGGFRRGKMTGHDVDFLITSPEATEDEEQQLLHKVTDFWKQQGLLLYCDILESTFEKFKOPSRKVDALDHFOKCFLI LKLDHGRVHSEKSGQOEGKGWKAIRVDLVMCPYDRPA FALLGWTGSRQFERDLRRYATHERKMMLDNHALYDRTKRVELEAESEEEIFAHLGLDYIEPUERMA

MGHHHнHнHнHнSSGHI DDDDKHMMKIEEGKLVIWINGDKGYNGLAEVGKKFEKDTGIKVTVEHPDKLEEKF QQVAATGDGPDI IFWAHDRFGGYAQSGLLAEITPDKAFQDKLYPFTWDAVRYNGKLIAYPIAVEALSLIYNKDLLPNPPKTW EEIPALDKELKAKGKSALMFNLQEPYFTWPLIAADGGYAFKYENGKYDIKDVGVDNAGAKAGLTELVDLIKNKHMNADTDYSIAEAAFNKGETAMTINGPWAWSNIDTSKVNYGVTVLPTFRGQPSKPFVGVLSAG INAASPNKELAKEELENY

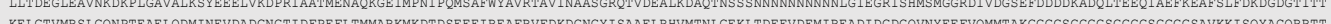
KE

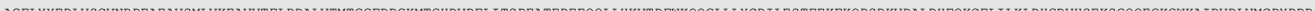
FALL

MBP-CaM-mTdT(M13-287)

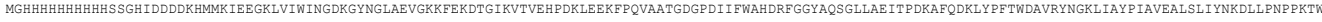

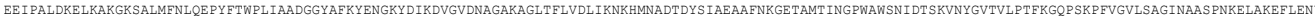
LLTDEGLEAVNKDKP LGAVALKSYEEELVRDPRIAATMENAQRGE IMPNI PQMSAFWYAVRTAVINAASGRQTVDEALKDAQTNSSSNWNWNWWNNNGIEGRISHMSMGGRDIVDGSEFDDDDKADQLTEEQIAEFKEAFSLFDKDGDGTIT

MBP-CaM-mTdT(M13-388) KELGTVMRSLGQNPTEAELQDMINEVDADGNGTIDFPEFLTMMARRMKDTDSEEEIREAFRVFDKDGNGYISAAELRHVMTNLGEKLTDEEVDEMIREADIDGDGQVNYEEFVQMMTAKGGGGSGGGGSGGGGSGGGGSAVKKI SQYACQRRT VKEAVVTFL PDALVTMTGG FRRGKMTGHDVDFLITS PEATEDEEQQLLHKVTDFWKQQGLLLYCDILESTEEKFGSARRKWQKTGHAVRAIGRLSSGSKQPSRKVDALDHFQKCFLILKLDHGRVHSEKSGQQEGKGWKAIRVDLVMMCPYDRR

Table S2: Amino acid sequences of TdT-CaM fusions

Expression vector sequences available upon request 